\title{
Chronic effects of a high sucrose diet on rodent small intestinal gene expression levels
}

\author{
A. Psichas, P. Sim, V. Preedy, T. Sanders, P. Taylor, L. Poston, M. Arno, \\ E. Aldecoa-Otalora and C. Corpe \\ Diabetes and Nutritional Sciences Division, School of Medicine, King's College London, Franklin-Wilkins Building, \\ London, SE1 9NH, UK
}

The global incidence of the metabolic syndrome is increasing at an alarming rate. However, the role of the gut in the development of dietinduced metabolic syndrome is poorly understood. The aim of this study was to determine the effects of a chronic high-sucrose diet (HSD) on gene expression levels in the small intestine of a mouse model of the metabolic syndrome ${ }^{(1)}$.

Sixteen C57BL/6J male mice were either fed on a $2 \%$ corn oil-pellet diet (control) or were given ad libitum access to $2 \%$ corn oil sucrose-sweetened milk (HSD) over a 6-week period. Animal tissues were isolated from the duodenum, jejunum and ileum. Microarrays were performed based on six groups of pooled RNA (duodenum/jejunum/ileum for control and HSD) extracted from the tissues.

On day 50 of the intervention, animals on the HSD were significantly more overweight compared with the control group $(P<0.001)$. In response to the chronic HSD, microarray analysis revealed 273, 319 and 503 differentially expressed genes in duodenum, jejunum, ileum, but no effect on the gene expression of sugar transporters, sweet taste receptors, gut peptides or gut peptide receptors $(v$. control). The HSD did result in a decrease in the mRNA expression of the glucose sensors SGLT3a, in the duodenum (2.6-fold), and SGLT3b in the duodenum and jejunum (4-2.6-fold). mRNA expression of the CD36 fat sensor was also down-regulated in the duodenum and jejunum (4-3-fold) in HSD-fed animals. mRNA expression of epithelial cell growth genes Gasdermin C3 and C4 were also up-regulated in the ileum (5-6-fold) in HSD-fed animals.

Microarray data indicate that at the gene expression level, small intestinal sugar transporters, sweet taste receptors and gut peptides appear to play no role in the development of HSD-induced metabolic syndrome. The potential roles of the glucose sensor, SGLT3, the fat sensor, CD36 and Gasdermin C in altered small intestinal function warrant further investigation.

1. Samuelsson AM, Matthews PA, Argenton M et al. (2008) Hypertension 51, 383-392. 